\title{
CATEGORIES ARISING FROM TABULAR ALGEBRAS
}

\author{
R. M. GREEN \\ Department of Mathematics and Statistics, Lancaster University, Lancaster LA1 4 YF, England \\ e-mail:r.m.green@lancaster.ac.uk
}

(Received 8 April, 2002; accepted 11 July, 2002)

\begin{abstract}
We continue the investigation of tabular algebras with trace (a certain class of associative $\mathbb{Z}\left[v, v^{-1}\right]$-algebras equipped with distinguished bases) by determining the extent to which the tabular structure may be recovered from a knowledge of the structure constants. This problem is equivalent to understanding a certain category (the category of table data associated to a tabular algebra) which we introduce. The main result is that this category is equivalent to another category (the category of based posets associated to a tabular algebra) whose structure we describe explicitly.
\end{abstract}

2000 Mathematics Subject Classification. 16B50.

Introduction. Tabular algebras with trace were introduced by the author in [4] as a class of algebras over the $\operatorname{ring} \mathcal{A}:=\mathbb{Z}\left[v, v^{-1}\right]$. They are by definition equipped with a tabular basis that is described in terms of a "table datum" and is required to satisfy various axioms. There are many natural examples of tabular bases, including the Kazhdan-Lusztig bases for certain Hecke algebras [8], the diagram bases of the Brauer algebra or Jones' annular algebra [7], and the IC bases of various kinds of TemperleyLieb algebra [5]. Tabular algebras also provide a convenient starting point from which to study cellular algebras in the sense of Graham and Lehrer [3]. Cellular algebras are of considerable interest in representation theory, and several constructions of cell data for specific algebras in the literature may be unified by using tabular algebras [4, Theorem 2.1.1]. Tabular algebras with trace are intriguing objects in their own right because all the natural examples (in fact, all the examples currently known to the author) have additional properties not required of them by the defining axioms, notably positivity of structure constants.

It is natural to wonder to what extent the table datum is determined by the tabular basis, particularly as the definition of a tabular algebra looks superficially complicated. More precisely, given a tabular algebra $A$ with distinguished basis $\mathbf{B}$ and trace $\tau$ satisfying axioms (A 1)-(A 5) (see $\S 2.1$ ), we wish to classify all possible table data compatible with the basis. A convenient way to do this is by using the technique of categorification, thus replacing the sets $A$ and $\mathbf{B}$ by a suitable category, $\mathcal{D}(A, \mathbf{B})$. The objects and morphisms of $\mathcal{D}(A, \mathbf{B})$ are defined in terms of the possible table data for $(A, \mathbf{B})$. Understanding the possible table data for $(A, \mathbf{B})$ is then equivalent to understanding the structure of the category $\mathcal{D}(A, \mathbf{B})$.

Our main result (Theorem 3.1.6) is that the category $\mathcal{D}(A, \mathbf{B})$ is equivalent to another category $\mathcal{P}(A, \mathbf{B})$, whose objects and morphisms can be easily and explicitly described in terms of "based posets", which we introduce. This solves the problem of understanding the structure of $\mathcal{D}(A, \mathbf{B})$. We also show that the algebra automorphisms 
of $A$ that fix $\mathbf{B}$ setwise may be understood in terms of these categories, and we show how these may be computed in typical cases.

Before we can define based posets and state the main results, it is necessary to develop some elementary theory regarding matrix rings over table algebras, and their automorphisms. This is the subject of $\S 1$. We recall the definition of tabular algebras from [4] in $\S 2$. In $\S 3$, we introduce and study based posets. In $\S 4$, we illustrate some of the ideas of this paper using the Brauer algebra as a worked example; the reader unfamiliar with tabular algebras may prefer to look at $\S 4.2$ before reading $\S 2$.

The results of this paper are interesting largely because of their applications to representation theory. For example, it is possible to define combinatorially a class of "standard modules" for a tabular algebra $(A, \mathbf{B})$ in terms of the table datum, and the results of this paper can be used to show that the class of modules so obtained depends only on the pair $(A, \mathbf{B})$, and not on the table datum chosen. Applications such as these will be explored in a sequel to this paper, where it will be shown that the extended affine Hecke algebra of type $\widetilde{A}_{n}$ equipped with its Kazhdan-Lusztig basis is a tabular algebra with trace, and that the standard modules agree with the geometrically defined standard modules appearing in the work of Lusztig [9].

\section{Based rings and their automorphism groups.}

1.1. Table algebras. We begin by recalling the definition of a table algebra, which is a generalization of the integral group ring of an arbitrary group.

Definition 1.1.1. A table algebra is a pair $(\Gamma, B)$, where $\Gamma$ is an associative unital $R$-algebra for some $\mathbb{Z} \leq R \leq \mathbb{C}$ and $B=\left\{b_{i}: i \in I\right\}$ is a distinguished basis for $\Gamma$ such that $1 \in B$, satisfying the following three axioms:

(T1) The structure constants of $\Gamma$ with respect to the basis $B$ lie in $\mathbb{R}^{+}$, the nonnegative real numbers.

(T2) There is an algebra anti-automorphism ${ }^{-}$of $\Gamma$ whose square is the identity and that has the property that $b_{i} \in B \Rightarrow \overline{b_{i}} \in B$. (We define $\bar{i}$ by the condition $\overline{b_{i}}=b_{i}$.)

(T3) Let $\kappa\left(b_{i}, a\right)$ be the coefficient of $b_{i}$ in $a \in \Gamma$. Then there is a function $g$ : $B \times B \rightarrow \mathbb{R}^{+}$satisfying

$$
\kappa\left(b_{m}, b_{i} b_{j}\right)=g\left(b_{i}, b_{m}\right) \kappa\left(b_{i}, b_{m} \overline{b_{j}}\right)
$$

where $g\left(b_{i}, b_{m}\right)$ is independent of $j$, for all $i, j, m$.

REMARK 1.1.2. Table algebras first appeared in the work of Arad and Blau [1] in the case where $\Gamma$ is commutative and $B$ is finite. All table algebras in this paper will be normalized, meaning that the structure constants of $\Gamma$ with respect to the basis $B$ will be (nonnegative) integers and the function $g$ in axiom (T3) sends all pairs of basis elements to 1. These conditions are reminiscent of Sunder's discrete hypergroups [11], and they are clearly satisfied when $B=G$ is any group and $\Gamma=\mathbb{Z} G$.

LEMMA 1.1.3. Let $(\Gamma, B)$ be a normalized table algebra.

(i) The linear map

$$
t: a \rightarrow \kappa(1, a)
$$

is a trace on $\Gamma$ (that is, $t(x y)=t(y x)$ for all $x, y \in \Gamma)$. 
(ii) Let $a=\sum_{i \in I} z_{i} b_{i}$ where $b_{i} \in B$ and $z_{i} \in \mathbb{Z}$. Then $t(a \bar{a})=1$ if and only if $a= \pm b_{i}$ for some $i$.

Proof. Part (i) is an easy consequence of axiom (T3), which shows that $\kappa\left(1, b_{i} b_{j}\right)=$ $\delta_{i \bar{j}}$. Using this observation, we see that $t(a \bar{a})=\sum_{i \in I} z_{i}^{2}$, which proves (ii).

Definition 1.1.4. Let $(\Gamma, B)$ be a normalized table algebra. A basis element $b \in B$ is said to be grouplike if $b \bar{b}=\bar{b} b=1$.

The notion of a grouplike element is similar to Arad and Blau's notion of an "irreducible" element, but this needs to be restated in our context so that we can deal with the case where $B$ is infinite.

\subsection{Based rings.}

Definition 1.2.1. A based ring is a pair $(A, B)$, where $A$ is a unital $\mathbb{Z}$-algebra with free $\mathbb{Z}$-basis $B$ and nonnegative structure constants. A homomorphism $\phi$ : $(A, B) \rightarrow\left(A^{\prime}, B^{\prime}\right)$ of based rings is a homomorphism of abstract $\mathbb{Z}$-algebras $\phi: A \rightarrow A^{\prime}$ such that $\phi(b) \in B^{\prime} \cup\{0\}$ for all $b \in B$. Isomorphisms, automorphisms, etc. of based rings are defined analogously.

Clearly normalized table algebras are examples of based rings.

Lemma 1.2.2. Let $(\Gamma, B)$ be a normalized table algebra and let $b, g \in B$ with $b$ grouplike.

(i) We have bg $\in B$ and $g b \in B$.

(ii) The $\mathbb{Z}$-linear map sending $g^{\prime} \mapsto \bar{b} g^{\prime} b$ for all $g^{\prime} \in B$ is a based ring automorphism of $(\Gamma, B)$.

Proof. Claim (ii) is immediate from (i), since $\bar{b}$ is grouplike if and only if $b$ is, so it remains to prove (i).

Let $t$ be the trace of Lemma 1.1.3. We observe that

$$
t((g b)(\overline{g b}))=t(g b \bar{b} \bar{g})=t(g \bar{g})=1,
$$

where the second equality uses the fact that $b$ is grouplike, and the third equality uses Lemma 1.1.3 (ii). Lemma 1.1.3 (ii) and the assumption that $(\Gamma, B)$ is normalized show that $g b \in B$. To prove the other half of (i), note that $t((b g)(\overline{b g}))=t((\overline{b g})(b g))$ by Lemma 1.1.3 (i) and then proceed as before.

The main example of a based ring that is of interest for our purposes is the following.

Definition 1.2.3. Let $(\Gamma, B)$ be a normalized table algebra. The based ring $M_{n, \Gamma, B}$ is the ring of $n \times n$ matrices over the ring $\Gamma$, equipped with distinguished basis consisting of all elements $e_{i j} \otimes b$, where $e_{i j}$ is a matrix unit and $b \in B$.

We call the elements $e_{i i} \otimes 1$ (for $1 \leq i \leq n$ ) distinguished idempotents.

It is trivial to check that $M_{n, \Gamma, B}$ is indeed a based ring.

LEMMA 1.2.4. Let $(\Gamma, B)$ be a normalized table algebra, and let ${ }^{-}$be the table algebra anti-automorphism. Let $\alpha$ be an automorphism of $M_{n, \Gamma, B}$ (as a based ring).

(i) The map $\alpha$ sends distinguished idempotents of $M_{n, \Gamma, B}$ to distinguished idempotents. 
(ii) The $\mathbb{Z}$-linear map $*$ that sends $e_{i j} \otimes b$ to $\left(e_{i j} \otimes b\right)^{*}:=e_{j i} \otimes \bar{b}$ is an antiautomorphism of $M_{n, \Gamma, B}$ that commutes with $\alpha$.

(iii) If $M_{n^{\prime}, \Gamma^{\prime}, B^{\prime}}$ is a based ring with anti-automorphism $*^{\prime}$ (as in (ii)) and $\phi$ : $M_{n, \Gamma, B} \rightarrow M_{n^{\prime}, \Gamma^{\prime}, B^{\prime}}$ is an isomorphism of based rings, then $*^{\prime}=\phi \circ * \circ \phi^{-1}$.

Proof. To prove (i), we note that $\alpha$ preserves the identity element, which is expressed in terms of the based ring basis as

$$
1=\sum_{i=1}^{n}\left(e_{i i} \otimes 1\right) .
$$

As an automorphism of based rings, $\alpha$ permutes the basis elements, and (i) follows.

It is easy to check that the map $*$ is an anti-automorphism of based rings.

Consider two basis elements $e_{i j} \otimes b$ and $e_{k l} \otimes b^{\prime}$ of the based ring. The only way a distinguished idempotent can occur with nonzero coefficient in the product $\left(e_{i j} \otimes b\right)\left(e_{k l} \otimes b^{\prime}\right)$ is if $j=k, i=l$ and 1 occurs in the product $b b^{\prime}$. The last of these conditions happens if and only if $b^{\prime}=\bar{b}$ by axiom (T3). If all these conditions hold, we have $e_{k l} \otimes b^{\prime}=\left(e_{i j} \otimes b\right)^{*}$, and the only distinguished idempotent occurring in the product is $e_{i i} \otimes 1$, which occurs with coefficient 1 since $(\Gamma, B)$ is normalized. This characterizes $*$ in terms of the structure constants and distinguished idempotents, and (iii) follows.

Now consider the basis element $\alpha\left(e_{i j} \otimes b\right)$. Since $\alpha$ permutes the distinguished idempotents by (i), we may apply $\alpha$ to the equation

$$
\left(e_{i j} \otimes b\right)\left(e_{j i} \otimes \bar{b}\right)=\sum c_{p, q, b^{\prime}}\left(e_{p q} \otimes b^{\prime}\right)
$$

and argue as in the previous paragraph to show that

$$
\alpha\left(e_{j i} \otimes \bar{b}\right)=\alpha\left(\left(e_{i j} \otimes b\right)^{*}\right)=\left(\alpha\left(e_{i j} \otimes b\right)\right)^{*} .
$$

Claim (ii) follows by linearity.

1.3. Automorphisms of $M_{n, \Gamma, B}$. In $\S 1.3$, we take a closer look at the based rings $M_{n, \Gamma, B}$ of Definition 1.2.3. The following lemma shows how the based ring isomorphism type of such a ring is controlled by the data $n, \Gamma$ and $B$.

LEMMA 1.3.1. If $\alpha: M_{n, \Gamma, B} \rightarrow M_{n^{\prime}, \Gamma^{\prime}, B^{\prime}}$ is an isomorphism of based rings, then $n=n^{\prime}$ and $(\Gamma, B) \cong\left(\Gamma^{\prime}, B^{\prime}\right)$ as based rings.

Proof. Since $\alpha$ is an isomorphism of based rings, it induces a bijection between the bases of each based ring. Arguing as in the proof of Lemma 1.2.4 (i), we see that $\alpha$ sends distinguished idempotents to distinguished idempotents, so that in particular we have $n=n^{\prime}$.

Let $e$ be any distinguished idempotent in $M_{n, \Gamma, B}$ and let $\phi_{e}$ be the $\mathbb{Z}$-linear map from $(\Gamma, B)$ to $M_{n, \Gamma, B}$ for which $\phi_{e}(b)=e \otimes b$ for all $b \in B$. It is clear that $\phi_{e}$ is a monomorphism of based rings whose image is $e M_{n, \Gamma, B} e$, which shows that the isomorphism type of $(\Gamma, B)$ as a based ring is determined by that of $M_{n, \Gamma, B}$. The conclusion follows.

The classification of automorphisms of $M_{n, \Gamma, B}$ is more interesting than the proof of Lemma 1.3.1 suggests. This is due to the presence of what we call "twisted" 
isomorphisms (defined below) which may not send elements of the form $e_{i j} \otimes 1$ to elements of the form $e_{k l} \otimes 1$ if $i \neq j$.

Definition 1.3.2. Let $\alpha: M_{n, \Gamma, B} \rightarrow M_{n^{\prime}, \Gamma^{\prime}, B^{\prime}}$ be a homomorphism of based rings. If there exist a map $\sigma:\{1, \ldots, n\} \rightarrow\left\{1, \ldots, n^{\prime}\right\}$ and a homomorphism of based rings $\psi$ : $(\Gamma, B) \rightarrow\left(\Gamma^{\prime}, B^{\prime}\right)$ such that $\alpha\left(e_{i j} \otimes b\right)=e_{\sigma(i) \sigma(j)} \otimes \psi(b)$ for all $1 \leq i, j \leq n$ and $b \in B$ then we call $\alpha$ an untwisted homomorphism. Otherwise, we call $\alpha$ a twisted homomorphism.

Lemma 1.3.1 has the following.

COROLlary 1.3.3. If $M_{n, \Gamma, B}$ and $M_{n^{\prime}, \Gamma^{\prime}, B^{\prime}}$ are isomorphic as based rings, then they are isomorphic by an untwisted isomorphism.

Definition 1.3.4. Consider the based ring $M_{n, \Gamma, B}$. We associate to the sequence $\left(b_{1}, b_{2}, \ldots, b_{n}\right)$ of grouplike elements of $B$ the based ring automorphism $\beta=$ $\beta\left(b_{1}, b_{2}, \ldots, b_{n}\right)$ of $M_{n, \Gamma, B}$. This is defined to send the element $X \in M_{n, \Gamma, B}$ to $G^{-1} X G$, where

$$
G=\sum_{i=1}^{n} e_{i i} \otimes b_{i} .
$$

(This makes sense by Lemma 1.2.2.) The automorphism $\beta$ will be twisted unless all the $b_{i}$ are equal.

If $w$ is a permutation in the symmetric group $\mathcal{S}(n)$, we define an untwisted based ring automorphism, $\sigma_{w}$, of $M_{n, \Gamma, B}$ that sends $e_{i j} \otimes b$ to $e_{w^{-1} i, w^{-1} j} \otimes b$.

If $\varepsilon$ is a based ring automorphism of $(\Gamma, B)$ (not necessarily one of the form given in Lemma 1.2.2 (ii)), we define an untwisted based ring automorphism $\psi_{\varepsilon}$ of $M_{n, \Gamma, B}$ by $\psi_{\varepsilon}\left(e_{i j} \otimes b\right)=e_{i j} \otimes \varepsilon(b)$.

Proposition 1.3.5. The group $\operatorname{Aut}_{B}\left(M_{n, \Gamma, B}\right)$ of based ring automorphisms of $M_{n, \Gamma, B}$ is generated by the automorphisms of the form $\beta\left(b_{1}, b_{2}, \ldots, b_{n}\right), \sigma_{w}$ and $\psi_{\varepsilon}$ as given in Definition 1.3.4.

Proof. Let $\alpha$ be such an automorphism. By Lemma 1.2.4 (i), $\alpha$ permutes the distinguished idempotents, so by applying a suitable automorphism $\sigma_{w}$, we may assume without loss of generality that $\alpha\left(e_{i i} \otimes 1\right)=e_{i i} \otimes 1$ for all $1 \leq i \leq n$.

With the above assumption, it follows that for any $1 \leq k, l \leq n$ and $b \in B, \alpha\left(e_{k l} \otimes\right.$ $b)$ is of the form $e_{k l} \otimes b^{\prime}$. This is because

$$
\left(e_{k k} \otimes 1\right)\left(e_{k l} \otimes g\right)\left(e_{l l} \otimes 1\right)=e_{k l} \otimes g
$$

for any $g \in B$.

For $2 \leq i \leq n$, we define the element $b_{i}^{\prime} \in B$ by the condition $\alpha\left(e_{i, i-1} \otimes 1\right)=$ $e_{i, i-1} \otimes b_{i}^{\prime}$.

We claim that $b_{i}^{\prime}$ is grouplike. Recalling the map $*$ from Lemma 1.2.4 (ii), we note that $\left(e_{i, i-1} \otimes 1\right)\left(e_{i, i-1} \otimes 1\right)^{*}=e_{i i} \otimes 1$. Since $\alpha$ commutes with $*$ and fixes the distinguished idempotents, we must have $\left(e_{i, i-1} \otimes b_{i}^{\prime}\right)\left(e_{i-1, i} \otimes \bar{b}_{i}^{\prime}\right)=e_{i i} \otimes 1$, which implies that $b_{i}^{\prime} \bar{b}_{i}^{\prime}=1$. A similar argument establishes that $\bar{b}_{i}^{\prime} b_{i}^{\prime}=1$, so that $b_{i}^{\prime}$ is grouplike.

Let $b_{1}=1$ and define, for $2 \leq i \leq n, b_{i}:=b_{i}^{\prime} b_{i-1}^{\prime} \cdots b_{2}^{\prime}$. Let $\beta$ be the automorphism $\beta\left(b_{1}, b_{2}, \ldots, b_{n}\right)$ of Definition 1.3.4. (Note that $\beta$ commutes with $*$; this follows easily from the definition since the matrix $G$ in Definition 1.3.4 is diagonal.) A routine matrix calculation shows that $\beta\left(\alpha\left(e_{i, i-1} \otimes 1\right)\right)=e_{i, i-1} \otimes 1$ for all $2 \leq i \leq n$. Since $\beta$ and $\alpha$ 
commute with $*$, the map $\beta \alpha$ also fixes elements of the form $e_{i-1, i} \otimes 1$, and since $\beta \alpha$ is an algebra homomorphism, it fixes all elements of the form $e_{i j} \otimes 1$ for $1 \leq i, j \leq n$. We may therefore assume for the rest of the proof that $\alpha$ fixes all elements $e_{i j} \otimes 1$.

We now see that $\alpha$ is determined by its values on $e_{11} \otimes b$, because

$$
e_{i j} \otimes b=\left(e_{i 1} \otimes 1\right)\left(e_{11} \otimes b\right)\left(e_{1 j} \otimes 1\right) .
$$

By the second paragraph of the proof, $\alpha$ must send $e_{11} \otimes b$ to $e_{11} \otimes b^{\prime}$ for some $b^{\prime}$. As noted in the proof of Lemma 1.3.1, the $\mathbb{Z}$-linear map sending $b$ to $e_{11} \otimes b$ is a based ring monomorphism, so it follows that there is a based ring automorphism $\varepsilon$ of $(\Gamma, B)$ such that $\alpha\left(e_{11} \otimes b\right)=e_{11} \otimes \varepsilon(b)$. In other words, $\alpha$ is equal to $\psi_{\varepsilon}$, completing the proof.

2. Tabular algebras and their based rings. We now recall from [4] the definition of a tabular algebra and its associated table datum. The goal of this paper is to understand the extent to which the table datum is determined by the tabular basis.

2.1. Tabular algebras. We start by recalling the definition of the a-function, which is due to Lusztig.

Definition 2.1.1. Let $\mathcal{A}$ be the ring of Laurent polynomials $\mathbb{Z}\left[v, v^{-1}\right]$, let $A$ be an $\mathcal{A}$-algebra and let $\mathbf{B}$ be an $\mathcal{A}$-basis of $A$. For $X, Y, Z \in \mathbf{B}$, we define the structure constants $g_{X, Y, Z} \in \mathcal{A}$ by the formula

$$
X Y=\sum_{Z} g_{X, Y, Z} Z
$$

The a-function is defined by

$$
\mathbf{a}(Z)=\max _{X, Y \in \mathbf{B}} \operatorname{deg}\left(g_{X, Y, Z}\right)
$$

where the degree of a Laurent polynomial is taken to be the highest power of $v$ occurring with nonzero coefficient. We define $\gamma_{X, Y, Z} \in \mathbb{Z}$ to be the coefficient of $v^{\mathbf{a}(Z)}$ in $g_{X, Y, Z}$; this will be zero if the bound is not achieved.

Definition 2.1.2. A tabular algebra is an $\mathcal{A}$-algebra $A$, together with a table datum

$$
(\Lambda, \Gamma, B, M, C, *)
$$

satisfying axioms (A1)-(A3) below.

(A1) $\Lambda$ is a finite poset. For each $\lambda \in \Lambda,(\Gamma(\lambda), B(\lambda))$ is a normalized table algebra over $\mathbb{Z}$ and $M(\lambda)$ is a finite set. The map

$$
C: \coprod_{\lambda \in \Lambda}(M(\lambda) \times B(\lambda) \times M(\lambda)) \rightarrow A
$$

is injective with image an $\mathcal{A}$-basis of $A$. We assume that $\operatorname{Im}(C)$ contains a set of mutually orthogonal idempotents $\left\{1_{\varepsilon}: \varepsilon \in \mathcal{E}\right\}$ such that $A=\sum_{\varepsilon, \varepsilon^{\prime} \in \mathcal{E}}\left(1_{\varepsilon} A 1_{\varepsilon^{\prime}}\right)$ and such that for each $X \in \operatorname{Im}(C)$, we have $X=1_{\varepsilon} X 1_{\varepsilon^{\prime}}$ for some $\varepsilon, \varepsilon^{\prime} \in \mathcal{E}$. A basis arising in this way is called a tabular basis. 
(A2) If $\lambda \in \Lambda, S, T \in M(\lambda)$ and $b \in B(\lambda)$, we write $C(S, b, T)=C_{S, T}^{b} \in A$. Then * is an $\mathcal{A}$-linear involutory anti-automorphism of $A$ such that $\left(C_{S, T}^{b}\right)^{*}=C_{T, S}^{\bar{b}}$, where ${ }^{-}$ is the table algebra anti-automorphism of $(\Gamma(\lambda), B(\lambda))$. If $g \in \mathbb{C}(v) \otimes_{\mathbb{Z}} \Gamma(\lambda)$ is such that $g=\sum_{b_{i} \in B(\lambda)} c_{i} b_{i}$ for some scalars $c_{i}$ (possibly involving $v$ ), we write $C_{S, T}^{g} \in \mathbb{C}(v) \otimes_{\mathcal{A}} A$ as shorthand for $\sum_{b_{i} \in B(\lambda)} c_{i} C_{S, T}^{b_{i}}$. We write $\mathbf{c}_{\lambda}$ for the image under $C$ of $M(\lambda) \times B(\lambda) \times$ $M(\lambda)$.

(A3) If $\lambda \in \Lambda, g \in \Gamma(\lambda)$ and $S, T \in M(\lambda)$ then for all $a \in A$ we have

$$
\text { a. } C_{S, T}^{g} \equiv \sum_{S^{\prime} \in M(\lambda)} C_{S^{\prime}, T}^{r_{a}\left(S^{\prime}, S\right) g} \bmod A(<\lambda)
$$

where $r_{a}\left(S^{\prime}, S\right) \in \Gamma(\lambda)\left[v, v^{-1}\right]=\mathcal{A} \otimes_{\mathbb{Z}} \Gamma(\lambda)$ is independent of $T$ and of $g$ and $A(<\lambda)$ is the $\mathcal{A}$-submodule of $A$ generated by the set $\bigcup_{\mu<\lambda} \mathbf{c}_{\mu}$.

A tabular algebra with trace is a tabular algebra that also satisfies conditions (A4) and (A5) below.

(A4) Let $K=C_{S, T}^{b}, K^{\prime}=C_{U, V}^{b^{\prime}}$ and $K^{\prime \prime}=C_{X, Y}^{b^{\prime \prime}}$ lie in $\operatorname{Im}(C)$. Then the maximum bound for $\operatorname{deg}\left(g_{K, K^{\prime}, K^{\prime \prime}}\right)$ in Definition 2.1.1 is achieved if and only if $X=S, T=U$, $Y=V$ and $\kappa\left(b^{\prime \prime}, b b^{\prime}\right) \neq 0$ (where $\kappa$ is as in axiom (T3)). If these conditions all hold and furthermore $b=b^{\prime}=b^{\prime \prime}=1$, we require $\gamma_{K, K^{\prime}, K^{\prime \prime}}=1$.

(A5) There exists an $\mathcal{A}$-linear function $\tau: A \rightarrow \mathcal{A}$ (the tabular trace), such that $\tau(x)=\tau\left(x^{*}\right)$ for all $x \in A$ and $\tau(x y)=\tau(y x)$ for all $x, y \in A$, that has the property that for every $\lambda \in \Lambda, S, T \in M(\lambda), b \in B(\lambda)$ and $X=C_{S, T}^{b}$, we have

$$
\tau\left(v^{\mathbf{a}(X)} X\right)= \begin{cases}1 \bmod v^{-1} \mathcal{A}^{-} & \text {if } S=T \text { and } b=1, \\ 0 \bmod v^{-1} \mathcal{A}^{-} & \text {otherwise. }\end{cases}
$$

Here, $\mathcal{A}^{-}:=\mathbb{Z}\left[v^{-1}\right]$. We call the elements $C_{S, S}^{1}$ distinguished involutions.

REMARK 2.1.3. The idempotent condition in axiom (A1) ensures that $\mathbf{a}(Z)$ is always defined.

Tabular algebras are so called because they are an amalgamation of table algebras and cellular algebras in the sense of Graham and Lehrer [3]. Axioms (A1)-(A3) are modelled on the axioms for a cellular algebra.

In this paper, we will only be concerned with tabular algebras with trace; this class of examples includes all the examples mentioned in the introduction. Our goal is to show that although the axioms in Definition 2.1.2 seem complicated, one can recover the table datum "up to isomorphism" (in a sense that will be made precise) from the structure constants of the tabular basis. Another way to state our aim is by the following question.

QUeStion 2.1.4. Given a tabular algebra $A$ with trace and with tabular basis $\mathbf{B}$, to what extent can we recover the map $C$ ?

Question 2.1.4 can be viewed as a question about categories, as we will explain in $\S 2.4$.

It is too much to hope to recover the table datum from the structure of $A$ as an abstract algebra, as can be seen from the following result.

THEOREM 2.1.5 (Hertweck [6]). There exist finite groups $G$ and $H$ with $G ¥ H$ and $\mathbb{Z} G \cong \mathbb{Z} H$ (so that $\mathcal{A} G=\mathcal{A} \otimes_{\mathbb{Z}} G \cong \mathcal{A} \otimes_{\mathbb{Z}} H=\mathcal{A} H$ ). 
The relevance to tabular algebras is as follows. Since $G$ is a group, $\mathcal{A} G$ is a tabular algebra with trace: take $\Lambda$ to consist of a single element $\lambda, \Gamma(\lambda)=\mathbb{Z} G, B(\lambda)=G, M(\lambda)$ to be a single element $m, C(m, b, m)=b$ and $*$ to be the linear extension of inversion. We can take $\tau(x)$ to be the coefficient of the identity element in $x$. Hertweck's theorem then shows that the isomorphism type of $(\Gamma(\lambda), B(\lambda))$ as a based ring cannot be recovered from the isomorphism type of $\mathcal{A} G$ as an abstract algebra.

2.2. Based rings arising from tabular algebras. In [4, $\S 3]$, asymptotic versions of tabular algebras with trace are constructed, using methods from [10]. These asymptotic algebras are based rings in the sense of $\S 1.2$. They will be useful in answering Question 2.1.4 since it will turn out that we can recover information about the tabular algebra by studying the associated based ring.

Definition 2.2.1. Let $A$ be a tabular algebra with trace, and maintain the usual notation. Define $\widehat{X}:=v^{-\mathbf{a}(X)} X$ for any tabular basis element $X \in \operatorname{Im}(C)$. The free $\mathcal{A}^{-}$module $A_{\lambda}^{-}$is defined to be generated by the elements $\left\{\widehat{X}: X \in \mathbf{c}_{\lambda}\right\}$. We set $t_{X}$ to be the image of $\widehat{X}$ in

$$
A_{\lambda}^{\infty}:=\frac{A_{\lambda}^{-}}{v^{-1} A_{\lambda}^{-}} .
$$

The latter is a $\mathbb{Z}$-algebra with basis $\left\{t_{X}: X \in \mathbf{c}_{\lambda}\right\}$ and structure constants

$$
t_{X} t_{X^{\prime}}=\sum_{X^{\prime \prime} \in \mathbf{c}} \gamma_{X, X^{\prime}, X^{\prime \prime}} t_{X^{\prime \prime}}
$$

where the $\gamma_{X, X^{\prime}, X^{\prime \prime}} \in \mathbb{Z}$ are as in Definition 2.1.1. We also set

$$
A^{\infty}:=\bigoplus_{\lambda \in \Lambda} A_{\lambda}^{\infty}
$$

this is a $\mathbb{Z}$-algebra with basis $\left\{t_{X}: X \in \operatorname{Im}(C)\right\}$.

We will call the ring $A^{\infty}$ the based ring associated to the tabular algebra $A$. This terminology is justified by the following lemma.

LEMMA 2.2.2. Let A be a tabular algebra with trace, and maintain the usual notation.

(i) The algebra $A_{\lambda}^{\infty}$ with basis $\left\{t_{X}: X \in \mathbf{c}_{\lambda}\right\}$ is isomorphic as a based ring to $M_{|M(\lambda)|, \Gamma(\lambda), B(\lambda)}$. The isomorphism may be chosen to identify $t_{X}$ with $e_{S T} \otimes b$, where $X=C(S, b, T)$ and $M(\lambda)$ is identified with the set $\{1,2, \ldots,|M(\lambda)|\}$.

(ii) The algebra $A^{\infty}$ with basis $\left\{t_{X}: X \in \operatorname{Im}(C)\right\}$ is a based ring.

Proof. Part (i) follows from [4, Theorem 3.2.4 (i)] and its proof. Part (ii) is immediate from part (i) and the definition of $A^{\infty}$.

2.3. Reduced tabular algebras. It is clear from axiom (A3) of a tabular algebra that if $A$ is a tabular algebra with table datum $(\Lambda, \Gamma, B, M, C, *)$ then we may refine the partial order $\leq$ on $\Lambda$ to a larger partial order without disturbing any of the axioms. However, this extra freedom turns out to be inconvenient for our purposes in this paper since it obfuscates some of the symmetry properties of the cell datum. For this reason, we introduce the notion of a reduced tabular algebra, for which the partial order on $\Lambda$ 
is as small as possible. Most of our results concern reduced tabular algebras, but there is no loss of generality in assuming that a tabular algebra is reduced.

Definition 2.3.1. Let $A$ be a tabular algebra with table datum $(\Lambda, \Gamma, B, M, C, *)$, where $\Lambda$ is ordered by $\leq$. Let $\Lambda^{\prime}$ be a poset with the same underlying set as $\Lambda$, partially ordered by $\leq^{\prime}$, and write $\Lambda^{\prime} \preceq_{P} \Lambda$ if $\leq$ is a refinement of $\leq^{\prime}$. If $\left(\Lambda^{\prime}, \Gamma, B, M, C, *\right)$ is a table datum for $A$ for some $\Lambda^{\prime} \prec_{P} \Lambda$ then $A$ and its table datum are said to be non-reduced; otherwise, $A$ and its table datum are said to be reduced.

One of the advantages of reduced tabular algebras with trace is that the poset $\Lambda$ may be recovered up to isomorphism from the tabular basis.

Definition 2.3.2. Let $A$ be a tabular algebra. If $X$ and $X^{\prime}$ are tabular basis elements, we say that $X^{\prime} \preceq X$ if $X^{\prime}$ appears with nonzero coefficient in $K X K^{\prime}$ for some tabular basis elements $K, K^{\prime}$. The relation $\preceq$ on the tabular basis $\operatorname{Im}(C)$ is defined to be the transitive extension of this relation; it is reflexive by axiom (A1).

The following result is the first step towards recovering the table datum of a tabular algebra with trace from the structure constants.

Proposition 2.3.3. Let $A$ be a reduced tabular algebra with trace and table datum

$$
(\Lambda, \Gamma, B, M, C, *)
$$

Let $\preceq$ be as in Definition 2.3.2. Let $X=C_{S, T}^{b} \in \mathbf{c}_{\lambda}$ and $X^{\prime}=C_{U, V}^{b^{\prime}} \in \mathbf{c}_{\mu}$ be tabular basis elements (where $\lambda, \mu \in \Lambda$ and $\mathbf{c}_{\lambda}$ is as defined in axiom (A2)'). Then $X \preceq X^{\prime}$ if and only if $\lambda \leq \mu$, with $\lambda=\mu$ if and only if $X \preceq X^{\prime} \preceq X$. It follows that the tabular basis determines the sets $\mathbf{c}_{\lambda}$ and the isomorphism type of the poset $\Lambda$.

Proof. It is clear from axiom (A3), its mirror image under $*$ (see [4, Remark 1.3.2]) and the definition of $\preceq$ that $X \preceq X^{\prime}$ implies $\lambda \leq \mu$.

By [4, Proposition 3.1.3], we find that $\lambda=\mu$ (and thus $\mathbf{c}_{\lambda}=\mathbf{c}_{\mu}$ ) if and only if $X \preceq X^{\prime} \preceq X$. To complete the proof of the first assertion, it remains to show that if $\lambda<\mu$ then $X \preceq X^{\prime}$.

Assume $\lambda<\mu$. Since $A$ is reduced, the partial order $\leq$ is the smallest possible partial order compatible with axiom (A3). The fact that $\Lambda$ is finite means that there is a chain

$$
\lambda=\lambda_{1}<\lambda_{2}<\cdots<\lambda_{r}=\mu
$$

where, for each $1 \leq i<r$, there exist basis elements $Y_{i} \in \mathbf{c}_{\lambda_{i}}, Y_{i+1} \in \mathbf{c}_{\lambda_{i+1}}$ and $K \in \mathbf{B}$ such that $Y_{i}$ occurs with nonzero coefficient in the expansion of $K Y_{i+1}$. The idempotent condition of axiom (A1) shows that $Y_{i} \preceq Y_{i+1}$, and we have $Y_{1} \preceq Y_{r}$ by transitivity. The previous paragraph shows that $X \preceq Y_{1} \preceq X$ and $X^{\prime} \preceq Y_{r} \preceq X^{\prime}$, so that $X \preceq X^{\prime}$ as required.

The second assertion now follows from the observation that the definition of $\preceq$ depends only on the tabular basis and not on any details of the table datum.

COROLLARY 2.3.4. Let $A$ be a reduced tabular algebra with trace and let

$$
(\Lambda, \Gamma, B, M, C, *), \quad\left(\Lambda^{\prime}, \Gamma^{\prime}, B^{\prime}, M^{\prime}, C^{\prime}, *^{\prime}\right)
$$


be two table data for $A$ associated to the same tabular basis $\mathbf{B}=\operatorname{Im} C=\operatorname{Im} C^{\prime}$. Let $X \in \mathbf{B}$, and define $\lambda \in \Lambda$ and $\lambda^{\prime} \in \Lambda^{\prime}$ by the conditions $X \in \mathbf{c}_{\lambda}$ and $X \in \mathbf{c}_{\lambda^{\prime}}$. There is an isomorphism of based rings

$$
M_{|M(\lambda)|, \Gamma(\lambda), B(\lambda)} \cong M_{\left|M\left(\lambda^{\prime}\right)\right|, \Gamma\left(\lambda^{\prime}\right), B\left(\lambda^{\prime}\right)} .
$$

Proof. By Proposition 2.3.3, the set $\mathbf{c}_{\lambda}$ containing $X$ may be reconstructed from $X$ and the tabular basis. This enables us to recover $A_{\lambda}^{\infty}$ from Definition 2.2.1, and the conclusion follows from Lemma 2.2.2 (i).

2.4. Categories arising from table data. Question 2.1 .4 can be restated in terms of a certain category that we now introduce.

Definition 2.4.1. Let $A$ be a tabular algebra with trace $\tau$ and tabular basis $\mathbf{B}$. The category $\mathcal{D}(A, \mathbf{B})=\mathcal{D}(A, \mathbf{B}, \tau)$ is defined as follows.

Objects: All elements $(\Lambda, \Gamma, B, M, *)$ for which there exists $C$ such that

$$
(\Lambda, \Gamma, B, M, C, *)
$$

is a reduced table datum for $A$ with $\operatorname{Im}(C)=\mathbf{B}$.

Morphisms: Let $(\Lambda, \Gamma, B, M, *)$ and $\left(\Lambda^{\prime}, \Gamma^{\prime}, B^{\prime}, M^{\prime}, *^{\prime}\right)$ be objects of $\mathcal{D}(A, \mathbf{B})$, and fix $C$ such that $(\Lambda, \Gamma, B, M, C, *)$ is a reduced table datum for $A$ with $\operatorname{Im}(C)=\mathbf{B}$. The set of morphisms between $(\Lambda, \Gamma, B, M, *)$ and $\left(\Lambda^{\prime}, \Gamma^{\prime}, B^{\prime}, M^{\prime}, *^{\prime}\right)$ are the maps

$$
\left(C^{\prime}\right)^{-1} \circ C: \coprod_{\lambda \in \Lambda}(M(\lambda) \times B(\lambda) \times M(\lambda)) \rightarrow \coprod_{\lambda^{\prime} \in \Lambda^{\prime}}\left(M^{\prime}\left(\lambda^{\prime}\right) \times B^{\prime}\left(\lambda^{\prime}\right) \times M^{\prime}\left(\lambda^{\prime}\right)\right),
$$

where $C^{\prime}$ is such that $\left(\Lambda^{\prime}, \Gamma^{\prime}, B^{\prime}, M^{\prime}, C^{\prime}, *^{\prime}\right)$ is a reduced table datum for $A$ with $\operatorname{Im}\left(C^{\prime}\right)=$ $\mathbf{B}$, and composition is given by composition of maps.

REMARK 2.4.2. It is not clear at this stage that $\mathcal{D}(A, \mathbf{B})$ is a well-defined category, because it is not a priori obvious that the composition of two morphisms is another morphism or that the set of morphisms between two objects is independent of the choice of $C$. We will resolve this issue in Theorem 3.4.1.

Question 2.1.4 is asking for a classification of the morphisms from a given object in $\mathcal{D}(A, \mathbf{B})$. We will achieve this by exhibiting an equivalence of categories between $\mathcal{D}(A, \mathbf{B})$ and a category for which this question is easy to answer.

3. Based posets and their automorphisms. We now introduce the notion of a based poset, which allows us to state our main result, Theorem 3.1.6. Throughout $\S 3, A$ will be a reduced tabular algebra with trace $\tau$ and tabular basis $\mathbf{B}$.

\subsection{Based posets.}

Definition 3.1.1. A based poset is a triple $(\Lambda, \leq, f)$ where $(\Lambda, \leq)$ is a poset and $f$ is a function with the property that for each $\lambda \in \Lambda, f(\lambda)$ is a based ring. An isomorphism of based posets

$$
\alpha:(\Lambda, \leq, f) \rightarrow\left(\Pi, \leq^{\prime}, g\right)
$$

is an isomorphism of posets $\alpha:(\Lambda, \leq) \rightarrow\left(\Pi, \leq^{\prime}\right)$ such that for all $\lambda \in \Lambda, \alpha$ induces an isomorphism of based rings $\alpha: f(\lambda) \rightarrow g(\alpha(\lambda))$. 
Definition 3.1.2. Let $A$ be a reduced tabular algebra with trace and with tabular basis $\mathbf{B}$. Let $(\Lambda, \Gamma, B, M, C, *)$ be a table datum for $A$.

The based poset $P(A, \mathbf{B})=P(\Lambda, \Gamma, B, M, C, *)=P(\Lambda, \Gamma, B, M, *)$ associated to the table datum $(\Lambda, \Gamma, B, M, C, *)$ is the triple $(\Lambda, \leq, f)$ where $(\Lambda, \leq)$ is the poset in the table datum of $A$ and, for each $\lambda \in \Lambda, f(\lambda)$ is the based ring $M_{|M(\lambda)|, \Gamma(\lambda), B(\lambda)} \cong A_{\lambda}^{\infty}$. Such a based poset is equipped with an anti-automorphism, $*$, which by definition leaves the elements of the poset fixed and induces the map $*$ of Lemma 1.2.4 (ii) on each $f(\lambda)$.

The category $\mathcal{P}(A, \mathbf{B})$ has as objects all elements $P(D)$ for $D \in \mathcal{D}(A, \mathbf{B})$ (see Definition 2.4.1); the morphisms are isomorphisms of based posets.

DEFINITION 3.1.3. Let $A$ be a reduced tabular algebra with trace and tabular basis B. Let $D$ be an object of $\mathcal{D}(A, \mathbf{B})$ and let $P(D)$ be the corresponding object of $\mathcal{P}(A, \mathbf{B})$. A parametrization of $D$ consists of bijections

$$
s_{\lambda}: M(\lambda) \rightarrow\{1,2, \ldots,|M(\lambda)|\}
$$

and a map

$$
\pi: \coprod_{\lambda}(M(\lambda) \times B(\lambda) \times M(\lambda)) \rightarrow \coprod_{\lambda \in \Lambda} f(\lambda)
$$

such that for all $\lambda \in \Lambda, S, T \in M(\lambda)$ and $b \in B(\lambda), \pi(S, b, T)=e_{s_{\lambda}(S), s_{\lambda}(T)} \otimes b \in f(\lambda)$. We will typically refer to the parametrization $\left(\pi, \bigsqcup_{\lambda \in \Lambda} s_{\lambda}\right)$ as "the parametrization $\pi$ " for short.

Proposition 3.1.4. Let $A$ be a reduced tabular algebra with trace and tabular basis B. Let $D_{1}:=(\Lambda, \Gamma, B, M, *)$ and $D_{2}:=\left(\Lambda^{\prime}, \Gamma^{\prime}, B^{\prime}, M^{\prime}, *^{\prime}\right)$ be objects of $\mathcal{D}(A, \mathbf{B})$ with parametrizations $\pi_{1}$ and $\pi_{2}$ respectively. If there is a morphism $\theta: D_{1} \rightarrow D_{2}$ in $\mathcal{D}(A, \mathbf{B})$, then there is a morphism

$$
P(\theta): P\left(D_{1}\right):=(\Lambda, \leq, f) \rightarrow P\left(D_{2}\right):=\left(\Lambda^{\prime}, \leq^{\prime}, f^{\prime}\right)
$$

in $\mathcal{P}(A, \mathbf{B})$, depending on $\pi_{1}$ and $\pi_{2}$. In particular, the isomorphism type of $P(A, \mathbf{B})$ as a based poset is independent of the choice of table datum.

Proof. Proposition 2.3.3 shows that the sets $\mathbf{c}_{\lambda}$ are independent of the table datum, so that

$$
\theta(M(\lambda) \times B(\lambda) \times M(\lambda))=M^{\prime}\left(\lambda^{\prime}\right) \times B^{\prime}\left(\lambda^{\prime}\right) \times M^{\prime}\left(\lambda^{\prime}\right)
$$

for some $\lambda^{\prime}$ depending on $\lambda$. Since $A$ is reduced, Proposition 2.3.3 also shows that $\theta$ is compatible with the partial orders on the two table data, and thus that $\theta$ is a poset isomorphism.

We define the map $P(\theta): \coprod f(\lambda) \rightarrow \coprod g\left(\theta(\lambda)\right.$ ) (depending on $\pi_{1}$ and $\pi_{2}$ ) by $\mathbb{Z}$ linear extension of the condition that $P(\theta) \circ \pi_{1}=\pi_{2} \circ \theta$. The map $P(\lambda)$ respects the partitions induced by $\Lambda$ and $\Lambda^{\prime}$. It is an isomorphism of based posets by Corollary 2.3.4 and Lemma 2.2.2 (i), because it represents the identity map on $A^{\infty}$ with respect to certain bases. The conclusion follows.

The involution $*$ is respected by the map $P$ in the following sense. 
Lemma 3.1.5. Let $A$ and $\mathbf{B}$ be as in Proposition 3.1.4, and let $D:=(\Lambda, \Gamma, B, M, *)$ be an object of $\mathcal{D}(A, \mathbf{B})$. Then the involution $*$ determines and is determined by the maps * on the based poset $P(D)$ in $\mathcal{P}(A, \mathbf{B})$ given in Definition 3.1.2, and furthermore, the correspondence is independent of the parametrization chosen.

Proof. The map $*$ induces an obvious permutation of each set $M(\lambda) \times B(\lambda) \times$ $M(\lambda)$ for each $\lambda \in \Lambda$. Choose a parametrization $\pi$ of $D$ and define the map $P(*): \bigsqcup f(\lambda) \rightarrow \bigsqcup f(\lambda)$ by the condition $P(*) \circ \pi=\pi \circ *$. The map $P(*)$ is equal to the based poset anti-automorphism $*$ of Definition 3.1.2, and the map is independent of the parametrization chosen. The converse is easily checked: the map $*$ of $D$ may be reconstructed from the maps $*$ on the based posets $P(D)$, again independently of the choice of parametrization.

Because of Lemma 3.1.5, we may identify the map $*$ of $D$ with the antiisomorphism of the based poset $P(D)$, and we may denote them both by $*$.

Proposition 3.1.4 hints that $P$ may be a functor, which will turn out to be the case (see Theorem 3.4.1 (iv)). The raison d'être of based posets is the following result, which can be regarded as the main result of the paper and the answer to Question 2.1.4.

THEOREM 3.1.6. Let $A$ be a reduced tabular algebra with trace and with tabular basis B. The categories $\mathcal{D}(A, \mathbf{B})$ and $\mathcal{P}(A, \mathbf{B})$ are equivalent.

3.2. Classifying the isomorphisms of based posets. To understand the morphisms in the category $\mathcal{P}(A, \mathbf{B})$, we require the following definition.

Definition 3.2.1. Let $(\Lambda, \leq, f)$ and $\left(\Pi, \leq^{\prime}, g\right)$ be objects of $\mathcal{P}(A, \mathbf{B})$. Let $p_{\Lambda}$ : $\Lambda \rightarrow \Pi$ be an isomorphism of abstract posets, and for each $\lambda \in \Lambda$ let $p_{\lambda}$ be an untwisted isomorphism of based rings (see Definition 1.3.2) from $f(\lambda)$ to $g\left(p_{\Lambda}(\lambda)\right)$. We define the isomorphism of based posets

$$
\iota_{p}:(\Lambda, \leq, f) \rightarrow\left(\Pi, \leq^{\prime}, g\right)
$$

to be the isomorphism inducing the map $p_{\Lambda}$ on $\Lambda$ and the maps $p_{\lambda}$ on each $f(\lambda)$.

Let $(\Lambda, \leq, f)$ be an object of $\mathcal{P}(A, \mathbf{B})$, let $\lambda \in \Lambda$ and set $n=|M(\lambda)|$. Let $\beta\left(b_{1}, b_{2}, \ldots, b_{n}\right), \sigma_{w}$ and $\psi_{\varepsilon}$ be based ring automorphisms of $M_{n, \Gamma(\lambda), B(\lambda)}$. We define the based poset automorphism $\beta^{\lambda}\left(b_{1}, b_{2}, \ldots, b_{n}\right)$ (respectively, $\left.\sigma_{w}^{\lambda}, \psi_{\varepsilon}^{\lambda}\right)$ of $(\Lambda, \leq, f)$ to be the automorphism that induces the identity map on the underlying poset and on all based rings $f(\mu)$ for $\mu \neq \lambda$, and that induces the automorphism $\beta\left(b_{1}, b_{2}, \ldots, b_{n}\right)$ (respectively, $\sigma_{w}, \psi_{\varepsilon}$ ) on $f(\lambda)$.

REMARK 3.2.2. Definition 3.2.1 makes sense by Lemma 2.2.2 (i), which guarantees that the based rings involved are isomorphic to $M_{n, \Gamma, B}$ for suitable $n, \Gamma$ and $B$.

Proposition 3.2.3. Maintain the notation of Definition 3.2.1. Any morphism

$$
\alpha:(\Lambda, \leq, f) \rightarrow\left(\Pi, \leq^{\prime}, g\right)
$$

in $\mathcal{P}(A, \mathbf{B})$ can be expressed as a product of isomorphisms of the form $\iota_{p}$ and $\beta^{\lambda}\left(b_{1}, b_{2}, \ldots, b_{n}\right)$.

Proof. Corollary 1.3.3 reduces the problem to the case where $(\Lambda, \leq, f)=\left(\Pi, \leq^{\prime}, g\right)$, once we compose $\alpha$ with a suitable isomorphism $\iota_{p}$. The result now follows from Proposition 1.3.5, because the automorphisms $\sigma_{w}$ and $\psi_{\varepsilon}$ are untwisted. 
Proposition 3.2.4. Let $A$ be a tabular algebra with trace and tabular basis $\mathbf{B}$, and let

$$
\alpha:(\Lambda, \leq, f) \rightarrow\left(\Lambda^{\prime}, \leq^{\prime}, f^{\prime}\right)
$$

be a morphism in $\mathcal{P}(A, \mathbf{B})$. Then $\alpha$ intertwines the based poset anti-automorphisms of its source and target, and $\alpha$ takes distinguished idempotents to distinguished idempotents.

Proof. By Proposition 3.2.3, it is enough to verify this for $\alpha=\iota_{p}$ and $\alpha=$ $\beta^{\lambda}\left(b_{1}, b_{2}, \ldots, b_{n}\right)$. The first case follows easily from the definitions, and the second case is a consequence of Lemma 1.2.4.

3.3. Automorphisms of the tabular basis. It will turn out that morphisms in the category $\mathcal{D}(A, \mathbf{B})$ all arise from the following construction.

DeFinition 3.3.1. Let $A$ be a reduced tabular algebra with trace and tabular basis B. Let $D_{1}:=(\Lambda, \Gamma, B, M, *)$ and $D_{2}:=\left(\Lambda^{\prime}, \Gamma^{\prime}, B^{\prime}, M^{\prime}, *^{\prime}\right)$ be objects of $\mathcal{D}(A, \mathbf{B})$, with parametrizations $\pi_{1}$ and $\pi_{2}$ respectively.

Let $\alpha: P\left(D_{1}\right) \rightarrow P\left(D_{2}\right)$ be a morphism in $\mathcal{P}(A, \mathbf{B})$; such a morphism exists by Proposition 3.1.4. This induces a map

$$
\alpha\left(\pi_{1}, \pi_{2}\right): \coprod_{\lambda \in \Lambda}(M(\lambda) \times B(\lambda) \times M(\lambda)) \rightarrow \coprod_{\lambda^{\prime} \in \Lambda^{\prime}}\left(M^{\prime}\left(\lambda^{\prime}\right) \times B^{\prime}\left(\lambda^{\prime}\right) \times M^{\prime}\left(\lambda^{\prime}\right)\right)
$$

given by $\alpha\left(\pi_{1}, \pi_{2}\right)=\pi_{2}^{-1} \alpha \pi_{1}$. If maps $C$ and $C^{\prime}$ are chosen such that

$$
(\Lambda, \Gamma, B, M, C, *)
$$

and

$$
\left(\Lambda^{\prime}, \Gamma^{\prime}, B^{\prime}, M^{\prime}, C^{\prime}, *^{\prime}\right)
$$

are table data, $\alpha\left(\pi_{1}, \pi_{2}\right)$ induces a permutation $\alpha$ of $\mathbf{B}$ via

$$
\alpha:=C^{\prime} \circ \alpha\left(\pi_{1}, \pi_{2}\right) \circ C^{-1} .
$$

This may be extended $\mathcal{A}$-linearly to a map on $A$.

REMARK 3.3.2. It must be emphasised that the permutations of the tabular basis in Definition 3.3.1 are generally not algebra automorphisms of $A$.

Lemma 3.3.3. Maintain the notation of Definition 3.3.1, so that the morphism $\alpha$ in $\mathcal{P}(A, \mathbf{B})$ induces a permutation of $\mathbf{B}=\operatorname{Im}(C)=\operatorname{Im}\left(C^{\prime}\right)$. Then for each $\lambda \in \Lambda, \alpha$ induces a bijection from $M(\lambda)$ to $M^{\prime}(\alpha(\lambda))$ : if $S \in M(\lambda), \alpha(S)$ is defined by the condition $\alpha\left(\pi_{1}, \pi_{2}\right)(S, 1, S)=(\alpha(S), 1, \alpha(S))$. In particular, $\alpha$ fixes the distinguished involutions setwise.

Proof. The map $\alpha$ is compatible with the partitions of $\mathbf{B}$ by the sets $\Lambda$ and $\Lambda^{\prime}$ because it is a morphism of based posets. The bijections $\pi_{1} C^{-1}$ and $\pi_{2}\left(C^{\prime}\right)^{-1}$ send distinguished involutions to distinguished idempotents, so by Proposition 3.2.4 the permutation of $\mathbf{B}$ induced by $\alpha$ takes distinguished involutions to distinguished involutions. 
What is remarkable about Definition 3.3.1 is that the permutations of $\mathbf{B}$ arising are in fact morphisms in the category $\mathcal{D}(A, \mathbf{B})$, and that these morphisms can be made to act on the possible table data.

Proposition 3.3.4. Let $A$ be a reduced tabular algebra with trace and with tabular basis $\mathbf{B}$. Let $D_{1}=(\Lambda, \Gamma, B, M, *)$ and $D_{2}=\left(\Lambda^{\prime}, \Gamma^{\prime}, B^{\prime}, M^{\prime}, *^{\prime}\right)$ be objects of $\mathcal{D}(A, \mathbf{B})$ and let $C$ be a map such that $(\Lambda, \Gamma, B, M, C, *)$ is a table datum. Fix parametrizations $\pi_{1}$ and $\pi_{2}$ for $D$ and $D^{\prime}$ respectively. Let $\alpha$ be a morphism $P(D) \rightarrow P\left(D^{\prime}\right)$; this exists by Proposition 3.1.4. Then $\alpha\left(\pi_{1}, \pi_{2}\right)$ is a morphism from $D \rightarrow D^{\prime}$ in $\mathcal{D}(A, \mathbf{B})$ and $\left(\Lambda^{\prime}, \Gamma^{\prime}, B^{\prime}, M^{\prime}, \alpha(C), *^{\prime}\right)$ is another reduced table datum for $A$ (with respect to the same trace) where $\alpha(C):=C \circ \alpha\left(\pi_{1}, \pi_{2}\right)^{-1}$. Furthermore, $*$ and $*^{\prime}$ are equal as permutations of $\mathbf{B}$.

Proof. The last assertion follows from Lemma 1.2.4 (iii), Lemma 2.2.2 (i) and Lemma 3.1.5. For the other assertion, we check each of the five axioms.

A1. It is clear that $\alpha(C)$ is injective because $\alpha\left(\pi_{1}, \pi_{2}\right)$ is invertible and $C$ is injective. The other assertions follow easily from the definition of $\mathcal{P}(A, \mathbf{B})$.

A2. Proposition 3.2.4 and Lemma 3.1.5 show that $\alpha\left(\pi_{1}, \pi_{2}\right)^{-1} \circ *^{\prime}=* \circ$ $\alpha\left(\pi_{1}, \pi_{2}\right)^{-1}$. The map $C$ intertwines the maps $*$ on its domain and range by axiom (A2) applied to $C$. We therefore have

$$
\begin{aligned}
\left(C \circ \alpha\left(\pi_{1}, \pi_{2}\right)^{-1}(S, b, T)\right)^{*^{\prime}} & =\left(C \circ \alpha\left(\pi_{1}, \pi_{2}\right)^{-1}(S, b, T)\right)^{*} \\
& =C \circ\left(\left(\alpha\left(\pi_{1}, \pi_{2}\right)^{-1}(S, b, T)\right)^{*}\right) \\
& =C \circ\left(\alpha\left(\pi_{1}, \pi_{2}\right)^{-1}(S, b, T)^{*^{\prime}}\right) \\
& =C \circ \alpha\left(\pi_{1}, \pi_{2}\right)^{-1}(T, \bar{b}, S)
\end{aligned}
$$

as required.

A3. We note that $\alpha$, being an isomorphism of based posets, respects both the partition of $\mathbf{B}$ into subsets $\mathbf{c}_{\lambda}$ and the partial order on $\Lambda$. The claims regarding $A(<\lambda)$ follow from this. In particular, $\alpha$ induces a bijection of $\Lambda \rightarrow \Lambda^{\prime}$, also denoted by $\alpha$.

We now need to show the existence of a function $r_{a}^{\prime}$ with the required properties with respect to the candidate $(\Lambda, \Gamma, B, M, \alpha(C), *)$ for the cell datum. We write $r_{a}$ for the corresponding function associated to the original cell datum.

We need only check the cases $\alpha=\iota_{p}$ and $\alpha=\beta^{\lambda}\left(b_{1}, b_{2}, \ldots, b_{n}\right)$ by Proposition 3.2.3.

For $\alpha=\iota_{p}$ we have

$$
\alpha(C)(S, b, T)=C(\sigma(S), \psi(b), \sigma(T))
$$

for all $\lambda \in \Lambda^{\prime}, S, T \in M^{\prime}(\lambda)$ and $b \in B^{\prime}(\lambda)$. Here, $\sigma: M^{\prime}(\lambda) \rightarrow M\left(\alpha^{-1}(\lambda)\right)$ is the map given in Lemma 3.3.3 and $\psi:\left(\Gamma^{\prime}, B^{\prime}\right) \rightarrow(\Gamma, B)$ is the isomorphism of based rings determined by $p$. In this case, we define

$$
r_{a}^{\prime}\left(S^{\prime}, S\right):=\psi^{-1}\left(r_{a}\left(\sigma\left(S^{\prime}\right), \sigma(S)\right)\right)
$$

Axiom (A3) applied to $C$ now gives

$$
\begin{aligned}
a . C_{\sigma(S), \sigma(T)}^{\psi(b)} & =\sum_{\sigma\left(S^{\prime}\right) \in M\left(\alpha^{-1}(\lambda)\right)} C_{\sigma\left(S^{\prime}\right), \sigma(T)}^{r_{a}\left(\sigma\left(S^{\prime}\right), \sigma(S)\right) \psi(b)} \bmod A\left(<\alpha^{-1}(\lambda)\right) \\
& =\sum_{\sigma\left(S^{\prime}\right) \in M\left(\alpha^{-1}(\lambda)\right)} C_{\sigma\left(S^{\prime}\right), \sigma(T)}^{\psi\left(r_{(}^{\prime}\left(S^{\prime}, S\right) b\right)} \bmod A\left(<\alpha^{-1}(\lambda)\right) .
\end{aligned}
$$


This yields

$$
a . \alpha(C)_{S, T}^{b}=\sum_{S^{\prime} \in M^{\prime}(\lambda)} \alpha(C)_{S^{\prime}, T}^{r_{a}^{\prime}\left(S^{\prime}, S\right) b} \bmod A\left(<^{\prime} \lambda\right)
$$

and shows that the axiom holds with respect to the prospective table datum for $D^{\prime}$ and $r_{a}^{\prime}$ in place of $r_{a}$.

For the other case, we take $\alpha=\beta^{\lambda}\left(b_{1}, b_{2}, \ldots, b_{n}\right)$. In this case, $D=D^{\prime}$ and so $\pi_{1}=\pi_{2}=\pi$. For each $S \in M(\lambda)$, let us write $b_{S}$ for $b_{s_{\lambda}(S)}$, where $s_{\lambda}$ is associated to the parametrization $\pi$ in the usual way. In this case,

$$
\alpha(C)(S, b, T):=C\left(S, \overline{b_{S}} b b_{T}, T\right)
$$

(Recall that $\overline{b_{S}} b b_{T} \in B(\lambda)$ by Lemma 1.2 .2 because $b_{S}$ and $b_{T}$ are grouplike.) In this case, we set $r_{a}^{\prime}\left(S^{\prime}, S\right):=b_{S^{\prime}} r_{a}\left(S^{\prime}, S\right) \overline{b_{S}}$. Axiom (A3) applied to $C$ now gives

$$
\begin{aligned}
a . C_{S, T}^{\overline{b_{S}} b b_{T}} & =\sum_{S^{\prime} \in M(\lambda)} C_{S^{\prime}, T}^{r_{a}\left(S^{\prime}, S\right) \overline{b_{S}} b b_{T}} \bmod A(<(\lambda)) \\
& =\sum_{S^{\prime} \in M(\lambda)} C_{S^{\prime}, T}^{\overline{b_{S^{\prime}}^{\prime}}\left(S^{\prime}, S\right) b b_{T}} \bmod A(<(\lambda)) .
\end{aligned}
$$

This yields

$$
a . \alpha(C)_{S, T}^{b}=\sum_{S^{\prime} \in M^{\prime}(\lambda)} \alpha(C)_{S^{\prime}, T}^{r_{a}^{\prime}\left(S^{\prime}, S\right) b} \bmod A(<\lambda)
$$

as required.

A4. As in the verification of axiom (A3), we need only check the cases $\alpha=\iota_{p}$ and $\alpha=\beta^{\lambda}\left(b_{1}, b_{2}, \ldots, b_{n}\right)$. The case of $\alpha=\iota_{p}$ is a routine verification. For the other case, the condition for the degree bound to be achieved follows from the observation that

$$
\kappa\left(\overline{b_{S}} b^{\prime \prime} b_{V},\left(\overline{b_{S}} b b_{T}\right)\left(\overline{b_{T}} b^{\prime} b_{V}\right)\right)=\kappa\left(b^{\prime \prime}, b b^{\prime}\right) .
$$

The claim about the case $b=b^{\prime}=b^{\prime \prime}=1$ follows from Lemma 2.2.2 (i) and the fact that the product

$$
\left(e_{s_{\lambda}(S), s_{\lambda}(T)} \otimes \overline{b_{S}} b_{T}\right)\left(e_{s_{\lambda}(T), s_{\lambda}(V)} \otimes \overline{b_{T}} b_{V}\right)
$$

contains $e_{S_{\lambda}(S), s_{\lambda}(V)} \otimes \overline{b_{S}} b_{V}$ with coefficient 1 .

A5. The map $C \circ \alpha\left(\pi_{1}, \pi_{2}\right) \circ C^{-1}$ sends distinguished involutions to distinguished involutions by Lemma 3.3.3, and it commutes with the map $*=*^{\prime}$ on $\mathbf{B}$ by the argument establishing axiom (A2) above. Axiom (A5) follows easily from these observations.

3.4. Main results. We are now in a position to examine the category $\mathcal{D}(A, \mathbf{B})$.

THEOREM 3.4.1. Let $A$ be a reduced tabular algebra with trace and tabular basis B. Let $D_{1}:=(\Lambda, \Gamma, B, M, *)$ and $D_{2}:=\left(\Lambda^{\prime}, \Gamma^{\prime}, B^{\prime}, M^{\prime}, *^{\prime}\right)$ be objects of $\mathcal{D}(A, \mathbf{B})$, with parametrizations $\pi_{1}$ and $\pi_{2}$ respectively. 
(i) Let $\phi: P\left(D_{1}\right) \rightarrow P\left(D_{2}\right)$ be a morphism in $\mathcal{P}(A, \mathbf{B})$ and choose $C$ so that $(\Lambda, \Gamma, B, M, C, *)$ is a table datum. Then $\phi\left(\pi_{1}, \pi_{2}\right): D_{1} \rightarrow D_{2}$ is a morphism in $\mathcal{D}(A, \mathbf{B})$ and there exists $C^{\prime}$ such that $\left(\Lambda^{\prime}, \Gamma^{\prime}, B^{\prime}, M^{\prime}, C^{\prime}, *^{\prime}\right)$ is a cell datum and such that $\phi\left(\pi_{1}, \pi_{2}\right)=\left(C^{\prime}\right)^{-1} \circ C$.

(ii) Every morphism $\phi: D_{1} \rightarrow D_{2}$ in $\mathcal{D}(A, \mathbf{B})$ is of the form $\alpha\left(\pi_{1}, \pi_{2}\right)$ for some morphism $\alpha: P\left(D_{1}\right) \rightarrow P\left(D_{2}\right)$ in $\mathcal{P}(A, \mathbf{B})$.

(iii) The category $\mathcal{D}(A, \mathbf{B})$ is well defined.

(iv) The map $P$ is a functor from $\mathcal{D}(A, \mathbf{B})$ to $\mathcal{P}(A, \mathbf{B})$ (assuming each object of $\mathcal{D}(A, \mathbf{B})$ is assigned a parametrization $)$.

Proof. Part (i) is immediate from Proposition 3.3.4.

To prove (ii), we first fix such a $\phi$. Proposition 3.1.4 produces a morphism

$$
P(\phi): P\left(D_{1}\right) \rightarrow P\left(D_{2}\right)
$$

in $\mathcal{P}(A, \mathbf{B})$ depending on $\pi_{1}$ and $\pi_{2}$. Definition 3.3.1 then shows that $P(\phi)\left(\pi_{1}, \pi_{2}\right)=\phi$.

For (iii), let $\phi: D_{1} \rightarrow D_{2}$ be a morphism in $\mathcal{D}(A, \mathbf{B})$ and choose $C$ as in the statement of (i). By (ii), $\phi$ is of the form $\alpha\left(\pi_{1}, \pi_{2}\right)$ for some morphism $\alpha$ in $\mathcal{P}(A, \mathbf{B})$. Applying (i) to $\alpha$, we see that $\phi=\left(C^{\prime}\right)^{-1} \circ C$, where $C^{\prime}$ is such that $\left(\Lambda^{\prime}, \Gamma^{\prime}, B^{\prime}, M^{\prime}, C^{\prime}, *^{\prime}\right)$ is a cell datum. This shows that the morphisms defined in Definition 2.4.1 do not depend on the choice of $C$. It also shows that composition of morphisms is well-defined, because if $\phi=\left(C_{1}\right)^{-1} C_{2}$ and $\phi^{\prime}=\left(C_{3}\right)^{-1} C_{4}$ are morphisms in $\mathcal{D}(A, \mathbf{B})$, we may arrange for $C_{2}=C_{3}$ so that $\phi \circ \phi^{\prime}$ is a morphism. This proves (iii), and makes (iv) into an easy exercise.

We can now prove the main result.

Proof of Theorem 3.1.6. It is enough to prove that $P$ is an equivalence of categories.

Consider the full subcategory $\mathcal{D}^{\prime}(A, \mathbf{B})$ of $\mathcal{D}(A, \mathbf{B})$ whose objects are those $(\Lambda, \Gamma, B, M, *)$ for which each set $M(\lambda)$ consists of the first $|M(\lambda)|$ natural numbers. This object has a parametrization in which all the maps $s_{\lambda}$ are the identity. Furthermore, each object $X$ of $\mathcal{P}(A, \mathbf{B})$ arises as $P\left(D^{\prime}\right)$ for a unique $D^{\prime} \in \mathcal{D}^{\prime}(A, \mathbf{B})$. In this case, we define $Q(X):=D^{\prime}$. If $\alpha: X \rightarrow Y$ is a morphism in $\mathcal{P}(A, \mathbf{B})$, we define the morphism $Q(\alpha): Q(X) \rightarrow Q(Y)$ by $Q(\alpha)=\alpha(\pi, \pi)$, where $\pi$ is the identity parametrization. Theorem 3.4.1 shows that $Q$ is a functor and that $P Q$ is the identity functor on $\mathcal{P}(A, \mathbf{B})$.

Suppose all objects of $\mathcal{D}(A, \mathbf{B})$ have been assigned parametrizations. Let

$$
D_{1}:=(\Lambda, \Gamma, B, M, *)
$$

be an object of $\mathcal{D}(A, \mathbf{B})$. Let us write

$$
\left(\Lambda, \Gamma, B, M^{\prime}, *\right):=Q P\left(D_{1}\right)
$$

The parametrization $\pi_{1}$ of $D_{1}$ induces a morphism (i.e., an isomorphism) $\eta_{1}$ in $\mathcal{D}(A, \mathbf{B})$ from $D_{1}$ to $Q P\left(D_{1}\right)$ : this is the map that sends $(S, b, T)$ to $\left(s_{\lambda}(S), b, s_{\lambda}(T)\right)$ for each $\lambda \in \Lambda, S, T \in M(\lambda), b \in B(\lambda)$ and $s_{\lambda}(S), s_{\lambda}(T) \in M^{\prime}(\lambda)$. If $D_{2}$ is another object with $\phi$ : $D_{1} \rightarrow D_{2}$ a morphism in $\mathcal{D}(A, \mathbf{B})$, we then see that the maps $\eta$ give natural isomorphisms between the identity functor on $\mathcal{D}(A, \mathbf{B})$ and the functor $Q P$. Theorem 3.1.6 follows. 
4. Algebra automorphisms of tabular algebras. Recall that in Remark 3.3.2, we stated that permutations of the tabular basis arising from morphisms in $\mathcal{D}(A, \mathbf{B})$ do not always induce isomorphisms at the level of tabular algebras. However, the permutations of the tabular basis that do give algebra automorphisms may be understood using our results, and may be classified in natural examples. We do this for the Brauer algebra in $\S 4.2$. We do not claim that these results are original; they are provided merely to illustrate the results of this paper.

Proposition 4.1.1. Let $A$ be a reduced tabular algebra with trace and with tabular basis B. Let $(\Lambda, \Gamma, B, M, C, *)$ be a table datum for $A$. Let $\phi$ be an $\mathcal{A}$-algebra automorphism of $A$ preserving $\mathbf{B}$ setwise. Then $\phi$ is of the form $C \circ \theta \circ C^{-1}$ for some $\theta \in \operatorname{Aut}_{\mathcal{D}(A, \mathbf{B})}(D)$, where $D:=(\Lambda, \Gamma, B, M, *)$.

Proof. Since $\phi$ is an algebra automorphism preserving the tabular basis, it must (by Proposition 2.3.3) permute the collection of sets $\mathbf{c}_{\lambda}$, so $\phi$ induces a bijection $\alpha: \Lambda \rightarrow \Lambda$. This bijection is an isomorphism of posets because the tabular algebra is reduced and thus the partial order is determined by the algebra structure via axiom (A3). It is clear from the definition of a that $\mathbf{a}(\alpha(X))=\mathbf{a}(X)$ for all $X \in \operatorname{Im}(C)$. Corollary 2.3.4 shows that $\phi$ induces an isomorphism of based rings

$$
\alpha: \coprod_{\lambda} A_{\lambda}^{\infty} \rightarrow \coprod_{\lambda} A_{\lambda}^{\infty}
$$

If we choose a parametrization $\pi$ for $D$ and let $J$ be the map sending $X \in \mathbf{B}$ to $t_{X} \in A^{\infty}$, we find that

$$
\pi C^{-1} J^{-1}: \coprod A_{\lambda}^{\infty} \rightarrow \coprod M_{|M(\lambda)|, \Gamma(\lambda), B(\lambda)}
$$

extends to give an isomorphism between $P(D)$ and the based poset under consideration. The based poset isomorphism $\alpha$ thus gives a based poset isomorphism $\pi C^{-1} J^{-1} \alpha J C \pi^{-1} \in \operatorname{Aut}_{\mathcal{D}(A, \mathbf{B})}(P(D))$. Since $P$ is an equivalence of categories by the proof of Theorem 3.1.6, there is a morphism $\theta \in \operatorname{Aut}_{\mathcal{D}(A, \mathbf{B})}(D)$ such that $\theta=$ $C^{-1} J^{-1} \alpha J C$. The claim follows from the fact that $\phi=J^{-1} \alpha J$.

We can deduce the following result, which holds for any table datum.

COROllary 4.1.2. Let $A$ be a reduced tabular algebra with trace and with tabular basis $\mathbf{B}$. Let $\phi$ be an $\mathcal{A}$-algebra automorphism of A preserving $\mathbf{B}$ setwise. Then $\phi$ is a $*-$ automorphism (i.e., $\phi \circ *=* \circ \phi$ ) and $\phi$ takes distinguished involutions to distinguished involutions.

Proof. This follows from Proposition 4.1.1, Proposition 3.2.4 and the equivalence of categories given by Theorem 3.1.6.

4.2. The Brauer algebra. We now recall how Brauer's centralizer algebra (which we call "the Brauer algebra" for short) may be given the structure of a tabular algebra with trace and show how the results and techniques of this paper apply to it. We calculate the group of algebra automorphisms of the algebra that preserve the basis; many other natural examples of tabular algebras can be analysed similarly.

Some useful references on the Brauer algebra are [2], [3, $\S 4]$ and [12]. 


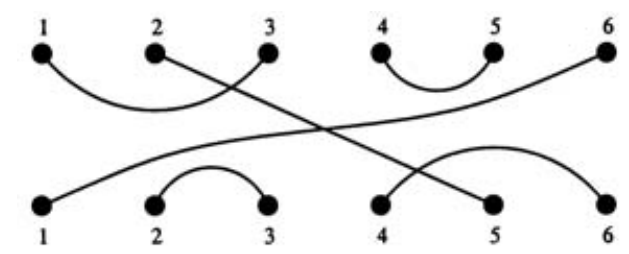

Figure 1. A Brauer algebra basis element for $n=6$.

Definition 4.2.1. The Brauer algebra $B_{n}(n \geq 2)$ is defined to be the unital associative $\mathcal{A}$-algebra with basis consisting of certain diagrams. A basis diagram, $D$, consists of two rows of $n$ points, labelled $\{1, \ldots, n\}$, with each point joined to precisely one point distinct from itself. (See Figure 1.)

Two diagrams $D_{1}, D_{2}$ may be composed to obtain $D_{1} \circ D_{2}$ by placing $D_{1}$ above $D_{2}$ and joining corresponding points. This produces a number $n\left(D_{1}, D_{2}\right)$ of interior loops, which are deleted. The product $D_{1} D_{2}$ in the Brauer algebra is defined by

$$
D_{1} D_{2}:=\left(v+v^{-1}\right)^{n\left(D_{1}, D_{2}\right)} D_{1} \circ D_{2} .
$$

As in $[3, \S 4]$, we may describe the basis diagrams in terms of certain triples.

Definition 4.2.2. Fix a diagram $D$. The integer $t(D)$ is defined to be the number of through strings, i.e., strings joining points in different rows. The involutions $S_{1}(D)$, $S_{2}(D)$ in the symmetric group $\mathcal{S}(n)$ are defined such that $S_{i}(D)$ interchanges the ends of the joins between points in row $i$, with $i \in\{1,2\}$. Corresponding to these we have subsets $\operatorname{Fix}\left(S_{i}(D)\right)$ of $\{1, \ldots, n\}$, which are the fixed points of the involutions $S_{i}(D)$. Finally, we have a permutation $w(D)$ in $\mathcal{S}(t)$, where $t=t(D)$; this is the permutation of Fix $\left(S_{1}(D)\right)$ determined by taking the end points of the through strings (regarded as joining from row 2 to row 1 ) in the order determined by taking their starting points in row 2 in increasing order. (We consider $\mathcal{S}(0)$ to be the trivial group, in which case $w$ is the identity.) The diagram $D$ is then determined by the triple $\left[S_{1}(D), S_{2}(D), w(D)\right]$.

We now recall a table datum for the Brauer algebra from [4, Example 2.1.2].

Definition 4.2.3. Let $B_{n}$ be the Brauer algebra (over $\mathcal{A}$ ) on $n$ strings. The algebra has a table datum $(\Lambda, \Gamma, B, M, C, *)$ as follows.

Take $\Lambda$ to be the set of integers $i$ between 0 and $n$ such that $n-i$ is even, ordered in the natural way. If $\lambda=0$, take $(\Gamma(\lambda), B(\lambda))$ to be the trivial one-dimensional table algebra; otherwise, take $\Gamma(\lambda)$ to be the group ring $\mathbb{Z} \mathcal{S}(\lambda)$ with basis $B(\lambda)=\mathcal{S}(\lambda)$ and involution $\bar{w}=w^{-1}$. Take $M(\lambda)$ to be the set of involutions on $n$ letters with $\lambda$ fixed points. Take $C\left(S_{1}, w, S_{2}\right)=\left[S_{1}, S_{2}, w\right] ; \operatorname{Im}(C)$ contains the identity element. The antiautomorphism $*$ sends $\left[S_{1}, S_{2}, w\right]$ to $\left[S_{2}, S_{1}, w^{-1}\right]$.

REMARK 4.2.4. There exists a tabular trace $\tau$ for $B_{n}$ (see [4, Remark 2.1.3]). One way to construct such a trace is to define $\tau(D)$ as follows on basis diagrams. Count the number, $k(D)$, of loops formed when each point $i$ in row 1 is joined to point $i$ in row 2 by a new string. Then the linear map $\tau: B_{n} \rightarrow \mathcal{A}$ such that $\tau(D):=v^{-n}\left(v+v^{-1}\right)^{k(D)}$ can be shown to be a trace with these properties. (We leave this as an exercise.)

Although $\Lambda$ is totally ordered in this case, it can easily be checked that the table datum given is reduced. 
The large supply of grouplike elements in the sets $B(\lambda)$ ensures that the corresponding based poset has plenty of symmetries, so by Theorem 3.4.1 (i), there are many choices for the map $C$ that give the same tabular basis of diagrams. As a consequence, there is nothing special about the diagrams parametrized by elements $C_{S, T}^{1}$; the set of diagrams that can be so expressed depends very much on the table datum. Another manifestation of this ambiguity is the fact that the definition of $w(D)$ depends on the choice of two orderings. However, the set of all diagrams of the form $\left[S_{1}(D), S_{1}(D), 1\right]$ does not depend on the choice of table datum by Lemma 3.3.3, because these are the distinguished involutions.

We wish to calculate the group of permutations of the diagram basis that preserve the algebra structure. Examples of such permutations are those which arise from relabelling the points $\{1, \ldots, n\}$ in rows 1 and 2 of each diagram by a fixed permutation in $\mathcal{S}(n)$. (Another way to think about this is to conjugate each diagram by a fixed diagram with $n$ through strings.) We will show that all basis preserving algebra automorphisms of $B_{n}$ are of this form; in particular, the outer automorphisms of the group $\mathcal{S}(6)$ do not extend to automorphisms of $B_{6}$. The elements $e_{a, b}$ and $g_{a, b}$ of the next definition will play a key role in the proof.

Definition 4.2.5. For $1 \leq a<b \leq n$, we define the basis elements $e_{a, b}$ and $g_{a, b}$ of $B_{n}$ as follows.

For the element $e_{a, b}$, point $j$ in row 1 is joined to point $j$ in row 2 , unless $j \in\{a, b\}$. Points $a$ and $b$ in row $i$ (for $i \in\{1,2\}$ ) are joined to each other.

For the element $g_{a, b}$, point $j$ in row 1 is joined to point $j$ in row 2 , unless $j \in\{a, b\}$. Point $a$ in row $i$ is joined to point $b$ in row $3-i$ (for $i \in\{1,2\}$ ).

It is clear that the elements $g_{a, b}$ generate a subalgebra of $B_{n}$ isomorphic to $\mathbb{Z}(\mathcal{S}(n))$, where $g_{a, b}$ corresponds to the transposition $(a, b)$. More importantly, we have the following well-known fact.

Proposition 4.2.6. The algebra $B_{n}$ is generated as a unital $\mathcal{A}$-algebra by the set $\left\{e_{k, k+1}: 1 \leq k<n\right\} \cup\left\{g_{k, k+1}: 1 \leq k<n\right\}$.

Proof. See [12, Proposition 2.1 (a)].

The elements $e_{a, b}$ and $g_{a, b}$ may be identified by the following properties which are independent of the table datum chosen. By Proposition 3.1.4, it makes sense to refer to the maximal element of the poset $\Lambda$ as $\lambda_{0}$, and to the second maximal element as $\lambda_{1}$. (Recall that $\Lambda$ is totally ordered, and that $n \geq 2$ so that $|\Lambda| \geq 2$.)

Lemma 4.2.7. The elements $e_{a, b}$ are precisely the distinguished involutions in the set $\mathbf{c}_{\lambda_{1}}$. The elements $g_{a, b}$ can be characterized as the only nonidentity elements in $\mathbf{c}_{\lambda_{0}}$ such that there exists a distinguished involution $e \in \mathbf{c}_{\lambda_{1}}$ with $g_{a, b} e=e$. (If this happens, we have $e=e_{a, b}$.) These characterizations depend only on the basis, and not on the table datum.

Proof. Axiom (A5) shows that the set of basis elements that are distinguished involutions is independent of the table datum. The other assertions follow easily from Definition 4.2.3.

We present the following result to illustrate our techniques and to confirm Remark 3.3.2. 
PROPOSITION 4.2.8. Let $\alpha: B_{n} \rightarrow B_{n}$ be an automorphism of $\mathcal{A}$-algebras preserving the diagram basis elements. Then there exists a diagram $X$ with $n$ through strings such that $\alpha(z)=X^{-1} z X$ for all $z \in B_{n}$.

Proof. By Proposition 4.1.1, $\alpha=C \circ \theta \circ C^{-1}$ for some $\theta \in \operatorname{Aut}_{\mathcal{D}(A, \mathbf{B})}(D)$, where $D=(\Lambda, \Gamma, B, M, *)$. By the main results, there is a corresponding automorphism $P(\theta)$ of the based poset $P(D)$ in $\mathcal{P}(A, \mathbf{B})$. Since $\Lambda$ is totally ordered, it has no non-trivial automorphisms as an abstract poset, so $P(\theta)$ fixes each poset element and $\alpha$ must fix $\mathbf{c}_{\lambda_{0}}$ and $\mathbf{c}_{\lambda_{1}}$ setwise. By Corollary 4.1.2 $\alpha$ permutes the distinguished involutions in the set $\mathbf{c}_{\lambda_{1}}$; in other words, for each $a$ and $b$ with $1 \leq a<b \leq n$ there exist $1 \leq c<d \leq n$ with $\alpha\left(e_{a, b}\right)=e_{c, d}$. By Lemma 4.2.7, we must have $\alpha\left(g_{a, b}\right)=g_{c, d}$. This determines $\alpha$ by Proposition 4.2.6. It remains to show that $\alpha$ is of the required form.

The map $\alpha$ induces an isomorphism of the quotient algebra $A / A\left(<\lambda_{0}\right)$ because it fixes each $\mathbf{c}_{\lambda}$ setwise. This algebra is naturally isomorphic to $\mathbb{Z} \mathcal{S}(n)$, and $\alpha$ induces an automorphism of $\mathcal{S}(n)$ that preserves cycle type. It follows that the action of $\alpha$ on $A / A\left(<\lambda_{0}\right)$ is effected by conjugation by an element $g \in \mathbf{c}_{0}$ (i.e., $\alpha(z)=g^{-1} z g$ ). The automorphism

$$
\phi: z \mapsto g \alpha(z) g^{-1}
$$

of $B_{n}$ preserves the diagram basis and fixes all the elements $g_{a, b}$. By Proposition 4.2.6 and Lemma 4.2.7, $\phi$ is the identity, which shows that $\alpha$ is conjugation by $g$ and completes the proof.

ACKNOWLEDGEMENT. The author is grateful to the referee for helpful suggestions.

\section{REFERENCES}

1. Z. Arad and H. I. Blau, On table algebras and applications to finite group theory, J. Algebra 138 (1991), 137-185.

2. R. Brauer, On algebras which are connected with the semisimple continuous groups, Ann. of Math. 38 (1937), 854-887.

3. J. J. Graham and G. I. Lehrer, Cellular algebras, Invent. Math. 123 (1996), 1-34. $27-64$.

4. R. M. Green, Tabular algebras and their asymptotic versions, J. Algebra 252 (2002),

5. R. M. Green and J. Losonczy, Canonical bases for Hecke algebra quotients, Math. Res. Lett. 6 (1999), 213-222.

6. M. Hertweck, A counterexample to the isomorphism problem for integral group rings, Ann. of Math. (2) 154 (2001), 115-138.

7. V. F. R. Jones, A quotient of the affine Hecke algebra in the Brauer algebra, Enseign Math (2) 40 (1994), 313-344.

8. D. Kazhdan and G. Lusztig, Representations of Coxeter groups and Hecke algebras, Invent. Math. 53 (1979), 165-184. $297-328$

9. G. Lusztig, Cells in affine Weyl groups IV, J. Fac. Sci, Tokyo Univ. (IA) 36 (1989),

10. G. Lusztig, Quantum groups at $v=\infty$, Prog. Math. 131 (1995), 199-221.

11. V. S. Sunder, $\mathrm{II}_{1}$ factors, their bimodules and hypergroups, Trans. Amer. Math. Soc. 330 (1992), 227-256

12. H. Wenzl, On the structure of Brauer's centralizer algebras, Ann. of Math. 128 (1988), $173-193$ 